\title{
SCATTERING OF ELECTROMAGNETIC WAVES BY THIN HIGH CONTRAST DIELECTRICS: EFFECTS OF THE OBJECT BOUNDARY*
}

\author{
DAVID M. AMBROSE ${ }^{\dagger}$ AND SHARI MOSKOW $\ddagger$
}

\begin{abstract}
We study the scattered field from a thin high contrast dielectric volume of finite extent. The waves are modeled by the full three dimensional time-harmonic Maxwell equations while accounting for material boundaries. We derive a formulation of Lippmann-Schwinger type for a dielectric scatterer; this formulation has an additional surface term to account for the material discontinuities. The layer potential operator resulting from this surface term is shown to converge in a weak sense to an explicitly computable limit as the thickness of the domain approaches zero. By properly accounting for the boundary effects, we show two results about the thin high contrast limit: First, the normal component of the electric field's interior trace on the lateral boundary approaches zero. Second, the third component of the electric field (which corresponds to the direction perpendicular to the slab) goes to zero inside the slab. We propose a new two-dimensional limiting equation as a first-order computational technique.
\end{abstract}

Key words. Scattering, Maxwell's equation, asymptotics, thin dielectric.

AMS subject classifications. 78A45, 45E99, 34E05.

\section{Introduction}

In electromagnetic scattering theory, volume integral formulations for Maxwell's equations are frequently very helpful for studying certain asymptotic regimes or inverse problems. However, these formulations can lead to difficulties dealing with material jump discontinuities, and a typical approach is to smooth out such discontinuities. Here we are motivated by the study of thin photonic band gap materials, in which the magnitude of such a jump may not lend itself to regularization. Furthermore, even when regularization is suitable, it can be quite difficult to take the limit as the regularization vanishes. Here we propose instead to start with a formulation for the full Maxwell's equations which takes the jumps into account. We then perform an asymptotic analysis which does not require regularization.

In the paper [9], Santosa, Zhang, and the second author proposed an approximate method to compute scattered fields from thin high contrast dielectric structures, using the Helmholtz equation. In this model, the squared index of refraction is assumed to be on the order of the reciprocal of the thickness of the structure, represented by a small parameter $h$. The method starts with the time-harmonic wave equations, and applies a perturbation approach based on expansions with respect to this small $h$. (We remind the reader that if the index of refraction were bounded the object would disappear in the limit.) This asymptotic approach was extended to Maxwell's equations in the presence of smoothly varying dielectrics in [1]. The advantage of these methods is that they reduce the complexity of the computation by one dimension (i.e., a threedimensional volume integral equation reduces to a set of two-dimensional integral equations). This leads to a highly efficient computational method for obtaining the scattered field anywhere in $\mathbb{R}^{3}$ : one can first inexpensively solve for the field using the

${ }^{*}$ Received: February 14, 2012; accepted (in revised form): May 9, 2012. Communicated by Richard Tsai.

${ }^{\dagger}$ Department of Mathematics, Drexel University, Philadelphia, PA 19104, USA (ambrose@ math.drexel.edu).

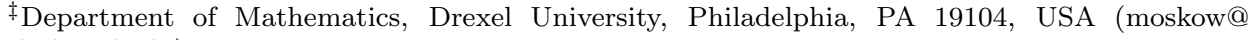
math.drexel.edu). 
dimensionally reduced system inside the scatterer, and then use this approximation as input into a Lippmann-Schwinger form. In the current paper, we study the same scattering problem, using the full three-dimensional Maxwell time-harmonic system, allowing for jumps in the material with respect to a constant background. We find that surface terms arising from material jumps do indeed impact the resulting system and approximations.

In our first theorem, we derive a generalized Lippmann-Schwinger equation for a Maxwell scatterer in the presence of jump discontinuities. The proof uses the Maxwell scattering formulations from [7] and [8], originally derived for factorization methods in inverse problems. In the absence of discontinuities, this formulation developed in the current work reduces to the Lippmann-Schwinger form studied in [1]. The generalized version has a surface integral on the jumps, unlike the original equation which has only volume integrals. The discontinuities that this formulation allows for can be from the external boundary of the scattering object and the background, or could just as well be from internal discontinuities such as a periodic array of air holes.

The new surface term corresponds to an integral operator that depends on our small parameter $h$. Correct asymptotic analysis will require that we understand the limiting behavior of this operator as the thickness of the domain vanishes. We compute the limit of this operator viewed on a fixed, scaled domain. The operators are of layer potential type, but converge to one that is instead algebraic in nature. This is what we show in our second theorem.

We restrict the electric field to its interior limit up to the object boundary using the integral formulation. In the high contrast case, we again take the limit as the thickness is going to zero, now with the dielectric constant simultaneously going to infinity. Under certain uniform regularity assumptions on the field, we show that the normal component of the field on the boundary of the object goes to zero. That is, in the limiting sense, the wave is trapped in the object. Next, we show that the third component of the electric field, which corresponds to the direction perpendicular to the scatterer, goes to zero inside of the scatterer. These two results about this high contrast media model are new, since this behavior was not observed in the models in [9] or [1]. Other previous work on thin dielectrics can be found in [2] and [10].

This paper is organized as follows. In Section 2, we give the precise setup for the thin high contrast dielectric scattering problem. We then derive the LippmannSchwinger form which we will use to analyze the problem, and prove that any regular enough solution to the differential equation satisfies it. Section 3 contains a study of the boundary integral operator which arises from the surface term on the jumps. The theorems about the trapping of the wave and the convergence of the perpendicular component are in Section 4. Finally, in Section 5 we discuss the implications of this work for asymptotic expansions and the related computational technique.

\section{Problem formulation}

2.1. Derivation. Let $E \in \mathbb{R}^{3}$ denote the electric field, which is governed by Maxwell's equations with a variable index of refraction and with the magnetic permeability, $\mu$, given by $\mu=1$. After eliminating the magnetic field this yields the following equation for $E$ :

$$
\nabla \times \nabla \times E-k^{2} n^{2}(x) E=0,
$$

where $k>0$ is the normalized frequency and $n^{2}(x)$ is the squared index of refraction. We assume that we have a cylindrical structure of small thickness $h$ on which the 
index of refraction is large. We incorporate this thin high contrast structure into the definition of $n^{2}(x)$ :

$$
n^{2}(x)=\left\{\begin{array}{lrl}
1 & \text { for }\left|x_{3}\right|>h / 2, \\
\varepsilon_{0}\left(x_{1}, x_{2}\right) / h \text { for }\left|x_{3}\right|<h / 2, & \left(x_{1}, x_{2}\right) \in \Omega, \\
1 & \text { for }\left|x_{3}\right|<h / 2, & \left(x_{1}, x_{2}\right) \notin \Omega,
\end{array}\right.
$$

where $\Omega$ is a bounded domain in $\mathbb{R}^{2}$ with smooth boundary. We denote the region of the dielectric object by

$$
\Omega_{h}=\Omega \times(-h / 2, h / 2) .
$$

We assume that $\varepsilon_{0}$ is smooth inside of $\Omega_{h}$, but here $n^{2}(x)$ has a jump discontinuity across $\partial \Omega_{h}$. Note that nowhere do we assume $\Omega$ is convex, and therefore the structure may contain holes whose boundaries are part of $\partial \Omega_{h}$.

The total field, $E$, can be written as $E=E_{\mathbf{i}}+E_{\mathbf{s}}$, where $E_{\mathbf{i}}$ is a given incident wave satisfying the background equation

$$
\nabla \times \nabla \times E_{\mathbf{i}}-k^{2} E_{\mathbf{i}}=0,
$$

and $E_{\mathbf{s}}$ is the scattered field which satisfies the Sommerfeld radiation condition. The $3 \times 3$ tensor

$$
G(x, y)=\phi(x, y) I+\frac{1}{k^{2}} \nabla_{y} \operatorname{div}_{y}(\phi(x, y) I)
$$

is the free space fundamental solution for the operator

$$
\nabla \times \nabla \times-k^{2}
$$

on $\mathbb{R}^{3}$, or the so-called dyadic Green's function. Here $\phi$ is the free space Helmholtz fundamental solution:

$$
\phi(x, y)=\frac{1}{4 \pi} \frac{e^{i k|x-y|}}{|x-y|} .
$$

With this dyadic Green's function we have the following Lippmann-Schwinger form for the equation for $E$ :

$$
E(x)=E_{\mathbf{i}}(x)+k^{2} \int_{\Omega_{h}} G(x, y) q(y) E(y) d y,
$$

where

$$
q(y)=n^{2}(y)-1=\frac{\varepsilon_{0}\left(y_{1}, y_{2}\right)}{h}-1 .
$$

Note that this form is a $3 \times 3$ system of integral equations for the three components of $E$. Plugging in the formula for $G$, we find

$$
E(x)=E_{\mathbf{i}}(x)+k^{2} \int_{\Omega_{h}} \phi(x, y) q(y) E(y) d y+\int_{\Omega_{h}} \nabla_{y} \operatorname{div}_{y}(\phi(x, y) I) q(y) E(y) d y .
$$

Let us assume, just for the time being, that the above form makes perfect sense despite the singular integral in the last term. Using the equation (2.1) for $E$, we can write $E$ as being proportional to a curl:

$$
E=\frac{\nabla \times \nabla \times E}{k^{2}(1+q(y))} .
$$


Using (2.8) and integrating by parts yields

$$
\begin{aligned}
& \int_{\Omega_{h}} \nabla_{y} \operatorname{div}_{y}(\phi(x, y) I) q(y) E(y) d y \\
= & \frac{1}{k^{2}} \int_{\Omega_{h}} \nabla_{y} \operatorname{div}_{y}(\phi(x, y) I) \frac{q(y)}{1+q(y)} \nabla \times \nabla \times E(y) d y \\
= & -\frac{1}{k^{2}} \int_{\Omega_{h}} \operatorname{div}_{y}(\phi(x, y) I) \nabla\left(\frac{q(y)}{1+q(y)}\right) \nabla \times \nabla \times E(y) d y \\
& \quad+\frac{1}{k^{2}} \int_{\partial \Omega_{h}} \operatorname{div}_{y}(\phi(x, y) I) \frac{q(y)}{1+q(y)}(\nabla \times \nabla \times E(y) \cdot \nu)^{-} d \sigma_{y} \\
= & -\int_{\Omega_{h}} \operatorname{div}_{y}(\phi(x, y) I) \nabla\left(\frac{q(y)}{1+q(y)}\right)(1+q(y)) E(y) d y \\
& \quad+\int_{\partial \Omega_{h}} \operatorname{div}_{y}(\phi(x, y) I) q(y)(E(y) \cdot \nu)^{-} d \sigma_{y},
\end{aligned}
$$

where we have used the fact that a curl of a gradient is zero. Here $\nu$ is the outward unit normal to $\partial \Omega_{h}$. A simple calculation shows that

$$
(1+q) \nabla\left(\frac{q}{1+q}\right)=\frac{\nabla q}{(1+q)} .
$$

Applying this in (2.7) yields

$$
\begin{array}{r}
E(x)=E_{\mathbf{i}}(x)+k^{2} \int_{\Omega_{h}} \phi(x, y) q(y) E(y) d y-\int_{\Omega_{h}} \operatorname{div}_{y}(\phi(x, y) I) \frac{\nabla q(y)}{1+q(y)} E(y) d y \\
+\int_{\partial \Omega_{h}} \operatorname{div}_{y}(\phi(x, y) I) q(y)(E(y) \cdot \nu)^{-} d \sigma_{y} .
\end{array}
$$

Since

$$
\operatorname{div}_{y}(\phi(x, y) I)=\nabla_{y} \phi(x, y)=-\nabla_{x} \phi(x, y),
$$

we have

$$
\begin{aligned}
E(x)= & E_{\mathbf{i}}(x)+k^{2} \int_{\Omega_{h}} \phi(x, y) q(y) E(y) d y \\
& +\nabla_{x} \int_{\Omega_{h}} \phi(x, y) \frac{\nabla q(y)}{1+q(y)} E(y) d y+\int_{\partial \Omega_{h}} \nabla_{y} \phi(x, y) q(y)(E(y) \cdot \nu)^{-} d \sigma_{y} .
\end{aligned}
$$

Note that without the last boundary term, this is precisely the formulation used in [1] for the case of smooth $n^{2}(x)$, which is derived from the Stratton-Chu formulas [3]. The last term appears here because there is a jump between the thin dielectric object and the homogeneous background. Replacing $q$ we have

$$
\begin{aligned}
E(x)=E_{\mathbf{i}}(x)+k^{2} \int_{\Omega_{h}} \phi(x, y)( & \left.\frac{\varepsilon_{0}(y)}{h}-1\right) E(y) d y+\nabla_{x} \int_{\Omega_{h}} \phi(x, y) \frac{\nabla \varepsilon_{0}(y)}{\varepsilon_{0}(y)} \cdot E(y) d y \\
& +\int_{\partial \Omega_{h}} \nabla_{y} \phi(x, y)\left(\frac{\varepsilon_{0}(y)}{h}-1\right)(E(y) \cdot \nu)^{-} d \sigma_{y} .
\end{aligned}
$$


Of the three integral kernel terms in the right hand side of the formulation for $E$ above, the first is the same that occurs for the special case of the Helmholtz equation, and its asymptotics with respect to $h$ were first analyzed in [9]. The second, as was previously mentioned, occurs in the formulation for Maxwell's equations for smooth $n \in \mathbb{R}^{3}$, and was first analyzed in [1]. Here we now take into account the jump between the scatterer and the background, and this leads to the final boundary integral term above. It is the analysis of this term and the resulting limiting equations for $E$ as $h \rightarrow 0$ that are the primary subject of the remaining sections of this paper.

2.2. Justification of integral formulation. Thanks to the statement of Theorem 9 in [8] (which was proved in [6]), we have that if $E \in H_{l o c}\left(\operatorname{curl}, \mathbb{R}^{3}\right.$ ) is a solution of (2.1) with the radiation conditions, then its restriction to $\Omega_{h}$ satisfies

$$
E(x)=E_{\mathbf{i}}(x)+k^{2} \int_{\Omega_{h}} \phi(x, y) q(y) E(y) d y+\nabla_{x} \operatorname{div}_{x} \int_{\Omega_{h}} \phi(x, y) q(y) E(y) d y .
$$

In fact, we also have from [6] that this integral equation has a unique solution in $H\left(\operatorname{curl}, \Omega_{h}\right)$, and this solution can be extended by the right hand side to a radiating solution of (2.1) in all of $\mathbb{R}^{3}$. Now, let us assume for simplicity that this solution $E$ is also smooth inside and up to the boundary $\partial \Omega_{h}$ from the interior (but not across $\left.\partial \Omega_{h}\right)$. Consider

$$
\operatorname{div}_{x} \int_{\Omega_{h}} \phi(x, y) q(y) E(y) d y=\int_{\Omega_{h}} \nabla_{x} \phi(x, y) \cdot E(y) q(y) d y,
$$

where we have interchanged integral and derivative since the kernel is only weakly singular. Since

$$
\nabla_{x} \phi(x, y)=-\nabla_{y} \phi(x, y)
$$

we integrate by parts to get

$$
\begin{aligned}
& \operatorname{div}_{x} \int_{\Omega_{h}} \phi(x, y) q(y) E(y) d y \\
= & -\int_{\Omega_{h}} \nabla_{y} \phi(x, y) \cdot E(y) q(y) d y \\
= & \int_{\Omega_{h}} \phi(x, y) \operatorname{div}(E(y) q(y)) d y-\int_{\partial \Omega_{h}} \phi(x, y) q(y)(E \cdot \nu)^{-} d \sigma_{y},
\end{aligned}
$$

where $(E \cdot \nu)^{-}$represents the limit on the boundary from the interior of $\Omega_{h}$. Using (2.8) and (2.9) and the fact the divergence of a curl is zero,

$$
\operatorname{div}(E(y) q(y))=\frac{\nabla q(y)}{1+q(y)} E(y)
$$

and hence

$$
\begin{aligned}
& \nabla_{x} \operatorname{div}_{x} \int_{\Omega_{h}} \phi(x, y) q(y) E(y) d y \\
= & \nabla_{x} \int_{\Omega_{h}} \phi(x, y) \frac{\nabla q(y)}{1+q(y)} E(y) d y-\nabla_{x} \int_{\partial \Omega_{h}} \phi(x, y) q(y)(E \cdot \nu)^{-} d \sigma_{y}
\end{aligned}
$$




$$
=\nabla_{x} \int_{\Omega_{h}} \phi(x, y) \frac{\nabla q(y)}{1+q(y)} E(y) d y+\int_{\partial \Omega_{h}} \nabla_{y} \phi(x, y) q(y)(E \cdot \nu)^{-} d \sigma_{y},
$$

where we have again interchanged derivative and integral and replaced the $x$ derivative with the negative of the derivative with respect to $y$. Note that for any $x$ in the interior of $\Omega_{h}$ the boundary integral is not singular and the interchange is completely justified. We have shown the following:

TheOREM 2.1. Let $E \in H_{\text {loc }}\left(\right.$ curl, $\left.\mathbb{R}^{3}\right)$ be the unique radiating solution to (2.1). Assume that we additionally have $E \in C^{0}\left(\overline{\Omega_{h}}\right) \cap H^{1}\left(\Omega_{h}\right)$. Then for interior $x \in \Omega_{h}, E(x)$ satisfies the integral equation

$$
\begin{aligned}
E(x)= & E_{\mathbf{i}}(x)+k^{2} \int_{\Omega_{h}} \phi(x, y)\left(n^{2}(y)-1\right) E(y) d y \\
& +\nabla_{x} \int_{\Omega_{h}} \phi(x, y) \frac{\nabla n^{2}(y)}{n^{2}(y)} \cdot E(y) d y+\int_{\partial \Omega_{h}} \nabla_{y} \phi(x, y)\left(n^{2}(y)-1\right)^{-}\left(E(y) \cdot \nu_{y}\right)^{-} d \sigma_{y} .
\end{aligned}
$$

Additionally, let $\nu$ be the outward unit normal to $\partial \Omega_{h}$, and $(E(x) \cdot \nu)^{-}$be the limit on the boundary from the interior of the normal component of $E$. Then, for $x \in \partial \Omega_{h}$, $(E(x) \cdot \nu)^{-}$satisfies

$$
\begin{aligned}
(E(x) \cdot \nu)^{-}= & E_{\mathbf{i}}(x) \cdot \nu+k^{2} \nu \cdot \int_{\Omega_{h}} \phi(x, y)\left(n^{2}(y)-1\right) E(y) d y \\
& +\nu \cdot \nabla_{x} \int_{\Omega_{h}} \phi(x, y) \frac{\nabla n^{2}(y)}{n^{2}(y)} \cdot E(y) d y+\frac{1}{2}\left(n^{2}(x)-1\right)^{-}(E(x) \cdot \nu)^{-} \\
& -\int_{\partial \Omega_{h}} \partial_{\nu_{x}} \phi(x, y)\left(n^{2}(y)-1\right)^{-}\left(E(y) \cdot \nu_{y}\right)^{-} d \sigma_{y} .
\end{aligned}
$$

The statement (2.17) follows from the fact that the normal component of the last term in (2.16) is the negative of a normal derivative of a single layer potential, and hence has a jump across the boundary of $\Omega_{h}$; see, for example, Theorem 3.28 of [4]. We also note that the representation (2.16) extends Theorem 9.1 of [3] to the case where $n^{2}(x)$ is piecewise smooth; the last term accounts for the jump in $n^{2}$ across $\partial \Omega_{h}$.

REMARK 2.1. Note that in the above theorem we need to assume that $E \in C^{0}\left(\overline{\Omega_{h}}\right)$. We expect that this will be the case for any fixed $h>0$, although we are not aware of a specific result to this effect. In future work the authors intend to make a detailed study of the regularity of solutions of Maxwell's equations for this geometry. This future study may also explore the extent to which we are able to relax assumptions on the domain, such as the smoothness of the boundary $\partial \Omega$. We also remark that the specific cylindrical geometry is not necessary for the above theorem; the theorem holds as long as $E$ satisfies the required regularity assumption inside the scatterer.

\section{The boundary layer potential operator and its limit}

Crucial to the above formulation (2.16) for Maxwell's equations is the last term on the right hand side, which involves $E \cdot \nu$ on the boundary of the scatterer. In order to correctly understand this term, we need the boundary formulation (2.17), which contains the surface operator

$$
-\int_{\partial \Omega_{h}} \partial_{\nu_{x}} \phi(x, y)\left(n^{2}(y)-1\right)^{-}\left(E(y) \cdot \nu_{y}\right)^{-} d \sigma_{y}
$$


We note that this term plays a crucial role for any scatterer, not just the high contrast model presented here. It has a nontrivial limit for thin domains, which we analyze in this section.

Consider the linear operator

$$
T_{h}^{*}: C^{0}\left(\partial \Omega_{h}\right) \rightarrow C^{0}\left(\partial \Omega_{h}\right)
$$

defined by

$$
\left(T_{h}^{*} f\right)(x)=\int_{\partial \Omega_{h}} \partial_{\nu_{x}} \phi(x, y) f(y) d \sigma_{y} .
$$

This is the dual of a double layer potential operator. We can show that when viewed on an appropriately scaled domain, the operators $T_{h}^{*}$ converge in a weak sense to an operator $T_{0}^{*}$. To be precise, define the scaled domain

$$
S=\Omega \times(-1 / 2,1 / 2)
$$

associated with the change of variables to $\tilde{y}$ given by

$$
y=\left(\tilde{y}_{1}, \tilde{y}_{2}, h \tilde{y}_{3}\right),
$$

and for any given $f(y)$, let

$$
\tilde{f}(\tilde{y})=f(y) .
$$

In what follows we will not want to require that functions are continuous at the corner curves of the pillbox, so we define the space

$$
C_{P}^{0}=C^{0}\left(\bar{\Omega} \times\left\{\frac{1}{2}\right\}\right) \oplus C^{0}\left(\bar{\Omega} \times\left\{-\frac{1}{2}\right\}\right) \oplus C^{0}\left(\partial \Omega \times\left[-\frac{1}{2}, \frac{1}{2}\right]\right)
$$

of piecewise continuous functions on $\partial S$. That is, $C_{P}^{0}$ is the space of functions which are separately continuous on the closures of the top, bottom, and lateral side of the scaled domain. Then define

by

$$
\tilde{T}_{h}^{*}: C_{P}^{0} \rightarrow C_{P}^{0}
$$

$$
\tilde{T}_{h}^{*} \tilde{f}=T_{h}^{*} f .
$$

Note that the elements of $C_{P}^{0}$ are not actually functions on $\partial S$ since they could be multiply defined on the corner curves $\partial \Omega \times\{1 / 2\}$ and $\partial \Omega \times\{-1 / 2\}$. To be precise, any $\tilde{f} \in C_{P}^{0}$ is a triple

$$
\tilde{f}=\left(f_{1}, f_{2}, f_{3}\right)
$$

where the first two components are functions on $\left(x_{1}, x_{2}\right) \in \bar{\Omega}$ (representing the top and bottom respectively), and the third component is a function on $\left(x_{1}, x_{2}, \tilde{x}_{3}\right) \in$ $\partial \Omega \times[-1 / 2,1 / 2]$ (representing the lateral side). For the sake of clarity of exposition, however, in what follows we sometimes abuse notation and identify elements of $C_{P}^{0}$ with functions on $\partial S$.

Given this notation, we can write 


$$
\begin{aligned}
&\left(\tilde{T}_{h}^{*} \tilde{f}\right)(\tilde{x})=\int_{\Omega \times\left\{\frac{1}{2}\right\}} \partial_{\nu_{x}} \phi(x, y) f_{1}(\tilde{y}) d \tilde{y}+\int_{\Omega \times\left\{-\frac{1}{2}\right\}} \partial_{\nu_{x}} \phi(x, y) f_{2}(\tilde{y}) d \tilde{y} \\
&+h \int_{\partial \Omega \times\left(-\frac{1}{2}, \frac{1}{2}\right)} \partial_{\nu_{x}} \phi(x, y) f_{3}(\tilde{y}) d \sigma_{\tilde{y}},
\end{aligned}
$$

where we note that the area element $d \tilde{y}=d y_{1} d y_{2}$ in the top and bottom pieces is unchanged in the new variables. In order to compute the limit of $\left\{\tilde{T}_{h}^{*}\right\}$ as $h$ approaches 0 , it is more straightforward to compute the limit of its $L^{2}$-based adjoint. The adjoint of $T_{h}^{*}$ is the complex conjugate of the usual double layer potential operator

$$
\left(T_{h} f\right)(x)=\int_{\partial \Omega_{h}} \partial_{\nu_{y}} \bar{\phi}(y, x) f(y) d \sigma_{y},
$$

which we also rescale to get

$$
\begin{aligned}
\left(\tilde{T}_{h} \tilde{f}\right)(\tilde{x})=\int_{\Omega \times\left\{\frac{1}{2}\right\}} \partial_{y_{3}} \bar{\phi}(y, x) f_{1}(\tilde{y}) d \tilde{y}- & \int_{\Omega \times\left\{-\frac{1}{2}\right\}} \partial_{y_{3}} \bar{\phi}(y, x) f_{2}(\tilde{y}) d \tilde{y} \\
& +h \int_{\partial \Omega \times\left(-\frac{1}{2}, \frac{1}{2}\right)} \partial_{\nu_{y}} \bar{\phi}(y, x) f_{3}(\tilde{y}) d \sigma_{\tilde{y}}
\end{aligned}
$$

While the true Banach space adjoint is defined on the larger dual space $\left(C_{P}^{0}\right)^{*}$, we consider its natural restriction to the piecewise continuous functions in $C_{P}^{0}$ and compute its weak limit.

TheOREM 3.1. The scaled double layer potential operators (3.6) defined on piecewise continuous functions on the boundary of the scaled domain $\partial S$,

$$
\tilde{T}_{h}: C_{P}^{0} \rightarrow C_{P}^{0},
$$

for $C_{P}^{0}$ defined by (3.3), converge pointwise weakly to a bounded operator

$$
\tilde{T}_{0}: C_{P}^{0} \rightarrow C_{P}^{0}
$$

in the sense that

$$
\left\langle\tilde{T}_{h} f, g\right\rangle \rightarrow\left\langle\tilde{T}_{0} f, g\right\rangle
$$

for any $f, g \in C_{P}^{0}$, where $\langle$,$\rangle is the L^{2}(\partial S)$ inner product. For a given $f \in C_{P}^{0}, \tilde{T}_{0} f$ is defined by

$$
\tilde{T}_{0}\left(\begin{array}{l}
f_{1} \\
f_{2} \\
f_{3}
\end{array}\right)=\left(\begin{array}{ccc}
0 & 1 / 2 & 0 \\
1 / 2 & 0 & 0 \\
-1 / 4 R & 1 / 4 R & 0
\end{array}\right)\left(\begin{array}{l}
f_{1} \\
f_{2} \\
f_{3}
\end{array}\right)
$$

where the operator

$$
R: C^{0}(\Omega) \rightarrow C^{0}(\partial \Omega \times(-1 / 2,1 / 2))
$$

is a restriction operator to $\partial \Omega$ followed by a constant extension in the $\tilde{x}_{3}$ direction.

Proof. We will complete the proof in two steps. First, we will demonstrate that $\tilde{T}_{h} f$ has the desired limit, pointwise in $x$. Second, we will demonstrate the weak convergence. 
Step 1: Pointwise convergence of $\tilde{T}_{h} f$ : Let us assume first that $\left(x_{1}, x_{2}\right) \in \Omega \backslash \partial \Omega$ and $x_{3}=h / 2,\left(\tilde{x}_{3}=1 / 2\right)$. That is, we will first find the limit for $\left(\tilde{T}_{h} \tilde{f}\right)$ restricted to the interior top portion of the scaled domain. In what follows we may use either $x_{3}$ or $h / 2$, but they are always equal in this part of the calculation.

We begin with (3.6). Since $\left(x_{1}, x_{2}\right)$ is away from the lateral side, the last term is not singular and goes to zero $O(h)$. Hence

$$
\begin{aligned}
\left(\tilde{T}_{h} \tilde{f}\right)(\tilde{x})=\int_{\Omega} \frac{\partial \bar{\phi}}{\partial y_{3}}\left(y_{1}, y_{2}, h / 2, x_{1}, x_{2}, h / 2\right) f_{1}(y) d y_{1} d y_{2} \\
\quad-\int_{\Omega} \frac{\partial \bar{\phi}}{\partial y_{3}}\left(y_{1}, y_{2},-h / 2, x_{1}, x_{2}, h / 2\right) f_{2}(y) d y_{1} d y_{2}+O(h) .
\end{aligned}
$$

Notice that for $x_{3}=y_{3}$, we have $\frac{\partial \bar{\phi}}{\partial y_{3}}(x, y)=0$; this implies that the first integral is identically zero. With the approximation $f_{2}\left(y_{1}, y_{2}\right) \approx f_{2}\left(x_{1}, x_{2}\right)$ in mind, we define the remainder term $R_{1}$ by the following equation:

$$
\left(\tilde{T}_{h} f\right)(\tilde{x})=-f_{2}\left(x_{1}, x_{2}\right) \int_{\Omega} \frac{\partial \bar{\phi}}{\partial y_{3}}\left(y_{1}, y_{2},-h / 2, x_{1}, x_{2}, h / 2\right) d \sigma_{y}+R_{1} .
$$

To see that the remainder $R_{1}$ goes to zero with $h$ for continuous $f_{2}$, consider

$$
\frac{\partial \bar{\phi}}{\partial y_{3}}=-\frac{1}{4 \pi} \frac{\partial}{\partial y_{3}}\left(\frac{e^{-i k|x-y|}}{\left(\left(x_{1}-y_{1}\right)^{2}+\left(x_{2}-y_{2}\right)^{2}+\left(\frac{h}{2}-h \tilde{y}_{3}\right)^{2}\right)^{1 / 2}}\right) .
$$

Since

$$
e^{-i k|x-y|} \approx 1-i k|x-y|
$$

we have that

$$
\left.\frac{\partial \bar{\phi}}{\partial y_{3}}\right|_{(y, x)}=-\frac{1}{4 \pi} \frac{x_{3}-h \tilde{y}_{3}}{\left(\left(x_{1}-y_{1}\right)^{2}+\left(x_{2}-y_{2}\right)^{2}+\left(\frac{h}{2}-h \tilde{y}_{3}\right)^{2}\right)^{3 / 2}}+O\left(\frac{x_{3}-h \tilde{y}_{3}}{|x-y|^{2}}\right) .
$$

Since $x_{3}=h / 2$, the remainder term will yield factors of $h$ multiplying kernels which are integrable in the limit as $h \rightarrow 0$. So, when we make the replacement $f_{2}\left(y_{1}, y_{2}\right) \approx$ $f_{2}\left(x_{1}, x_{2}\right)$, the remaining term is of the form

$$
\frac{1}{4 \pi} \int_{\Omega} \frac{h\left(f_{2}\left(x_{1}, x_{2}\right)-f_{2}\left(y_{1}, y_{2}\right)\right)}{\left(\left(x_{1}-y_{1}\right)^{2}+\left(x_{2}-y_{2}\right)^{2}+h^{2}\right)^{3 / 2}} d y_{1} d y_{2} .
$$

Now, if $f_{2}$ is a $C^{1}$ function, this integral clearly goes to zero with $h$ since in this case

$$
\left|f_{2}\left(x_{1}, x_{2}\right)-f_{2}\left(y_{1}, y_{2}\right)\right|=O(|x-y|)
$$

and the integrand is only weakly singular in the limit as $h \rightarrow 0$. So, let us approximate $f_{2}$ by a sequence of $C^{1}$ functions, $\left\{f_{2}^{n}\right\}$, that will converge to $f_{2}$ in the uniform norm as $n \rightarrow \infty$. Then, we write the following:

$$
\int_{\Omega} \frac{h\left(f_{2}(x)-f_{2}(y)\right)}{\left(\left(x_{1}-y_{1}\right)^{2}+\left(x_{2}-y_{2}\right)^{2}+h^{2}\right)^{3 / 2}} d y_{1} d y_{2}
$$




$$
\begin{aligned}
= & \left(f_{2}(x)-f_{2}^{n}(x)\right) \int_{\Omega} \frac{h}{\left(\left(x_{1}-y_{1}\right)^{2}+\left(x_{2}-y_{2}\right)^{2}+h^{2}\right)^{3 / 2}} d y_{1} d y_{2} \\
& +\int_{\Omega} \frac{h\left(f_{2}^{n}(x)-f_{2}^{n}(y)\right)}{\left(\left(x_{1}-y_{1}\right)^{2}+\left(x_{2}-y_{2}\right)^{2}+h^{2}\right)^{3 / 2}} d y_{1} d y_{2} \\
& +\int_{\Omega} \frac{h\left(f_{2}^{n}(y)-f_{2}(y)\right)}{\left(\left(x_{1}-y_{1}\right)^{2}+\left(x_{2}-y_{2}\right)^{2}+h^{2}\right)^{3 / 2}} d y_{1} d y_{2}=I+I I+I I I .
\end{aligned}
$$

One can check that by integrating around a small ball, the integral

$$
\int_{\Omega} \frac{h}{\left(\left(x_{1}-y_{1}\right)^{2}+\left(x_{2}-y_{2}\right)^{2}+h^{2}\right)^{3 / 2}} d y_{1} d y_{2}
$$

is bounded as $h \rightarrow 0$. So, for any $\epsilon>0$ we can choose $n$ large enough so that terms $I$ and $I I I$ are smaller than $\epsilon / 3$ for any $h \in(0,1]$. Then, since $f_{2}^{n}$ is $C^{1}$, we can choose $h$ small enough that $I I$ is also less than $\epsilon / 3$, so that the whole integral in (3.11) is less than $\epsilon$. This establishes that $R_{1}$ does go to zero when $h$ goes to zero, as claimed; furthermore, this convergence is uniform in $x$. We then have

$$
\left(\tilde{T}_{h} f\right)(\tilde{x})=\frac{1}{4 \pi} f_{2}\left(x_{1}, x_{2}\right) \int_{\Omega} \frac{x_{3}+h / 2}{\left(\left(x_{1}-y_{1}\right)^{2}+\left(x_{2}-y_{2}\right)^{2}+\left(\frac{h}{2}+\frac{h}{2}\right)^{2}\right)^{3 / 2}} d y_{1} d y_{2}+R_{2},
$$

where again $R_{2}$ goes to zero with $h$. Since $\left(x_{1}, x_{2}\right) \in \Omega \backslash \partial \Omega$, there exists $\delta>0$ such that the ball of radius $\delta$ centered at $\left(x_{1}, x_{2}\right), B_{\delta} \subseteq \Omega \backslash \partial \Omega$. We notice that outside of this ball, the integral will go to zero with $h$. We are then left with

$$
\left(\tilde{T}_{h} f\right)(\tilde{x})=\frac{1}{4 \pi} f_{2}\left(x_{1}, x_{2}\right) \int_{B_{\delta}} \frac{h}{\left(\left(x_{1}-y_{1}\right)^{2}+\left(x_{2}-y_{2}\right)^{2}+h^{2}\right)^{3 / 2}} d y_{1} d y_{2}+R_{3},
$$

where $R_{3}$ goes to zero with $h$. This integral can be calculated explicitly with polar co-ordinates. The result is

$$
\left(\tilde{T}_{h} \tilde{f}\right)(\tilde{x})=\left.f_{2}\left(x_{1}, x_{2}\right)(-h / 2)\left(r^{2}+h^{2}\right)^{-1 / 2}\right|_{r=0} ^{r=\delta}+R_{3} .
$$

We can evaluate this and include the $r=\delta$ contribution into the remainder, finding

$$
\left(\tilde{T}_{h} \tilde{f}\right)(\tilde{x})=\frac{f_{2}\left(x_{1}, x_{2}\right)}{2}+R_{4}
$$

where $R_{4}$ goes to zero with $h$. A similar calculation yields that for $x$ on the bottom in the interior of $\Omega,\left(x_{1}, x_{2}\right) \in \Omega \backslash \partial \Omega, x_{3}=-h / 2$,

$$
\left(\tilde{T}_{h} \tilde{f}\right)(\tilde{x})=\frac{f_{1}\left(x_{1}, x_{2}\right)}{2}+R_{5}
$$

where $R_{5}$ goes to zero with $h$.

Let us next consider $\tilde{x} \in \partial \Omega \times(-1 / 2,1 / 2)$. Although the last term in (3.4) has a singularity, it still goes to zero because of the factor of $h$ in front, and the fact that if $x$ and $y$ are both on the lateral boundary,

$$
(x-y) \cdot \nu_{y}=O\left(|x-y|^{2}\right)
$$


since $x-y$ is close to tangential when $x-y$ is small [4]. However, now neither the integral over the top nor the bottom is identically zero and both will contribute to the limit. Hence, we can write

$$
\begin{aligned}
& \left(\tilde{T}_{h} f\right)(\tilde{x}) \\
= & -\frac{1}{4 \pi} f_{1}\left(x_{1}, x_{2}\right) \int_{\Omega} \frac{x_{3}-h / 2}{\left(\left(x_{1}-y_{1}\right)^{2}+\left(x_{2}-y_{2}\right)^{2}+\left(x_{3}-h / 2\right)^{2}\right)^{3 / 2}} d y_{1} d y_{2} \\
& +\frac{1}{4 \pi} f_{2}\left(x_{1}, x_{2}\right) \int_{\Omega} \frac{x_{3}+h / 2}{\left(\left(x_{1}-y_{1}\right)^{2}+\left(x_{2}-y_{2}\right)^{2}+\left(x_{3}+h / 2\right)^{2}\right)^{3 / 2}} d y_{1} d y_{2}+R_{6} .
\end{aligned}
$$

Now, since $\left(x_{1}, x_{2}\right) \in \partial \Omega$, we can no longer take a ball contained in $\Omega$ about this point. However, for $\partial \Omega$ smooth, we can take a half-ball of radius $\delta$ about $\left(x_{1}, x_{2}\right)$ with flat side tangential to $\partial \Omega$; call this $H_{\delta}$. Introduction of this half-ball does introduce an error term unless the boundary is actually flat at $\left(x_{1}, x_{2}\right)$; however, this term can be made arbitrarily small by choosing $\delta$ small, since $\partial \Omega$ is smooth. The remainder of both of these integrals will go to zero with $h$ :

$$
\begin{aligned}
& \quad\left(\tilde{T}_{h} f\right)(\tilde{x}) \\
& =-\frac{1}{4 \pi} f_{1}\left(x_{1}, x_{2}\right) \int_{H_{\delta}} \frac{h\left(\tilde{x}_{3}-1 / 2\right)}{\left(\left(x_{1}-y_{1}\right)^{2}+\left(x_{2}-y_{2}\right)^{2}+\left(h\left(\tilde{x}_{3}-1 / 2\right)\right)^{2}\right)^{3 / 2}} d y_{1} d y_{2} \\
& \quad+\frac{1}{4 \pi} f_{2}\left(x_{1}, x_{2}\right) \int_{H_{\delta}} \frac{h\left(\tilde{x}_{3}+1 / 2\right)}{\left(\left(x_{1}-y_{1}\right)^{2}+\left(x_{2}-y_{2}\right)^{2}+\left(h\left(\tilde{x}_{3}+1 / 2\right)\right)^{2}\right)^{3 / 2}} d y_{1} d y_{2}+R_{7} .
\end{aligned}
$$

This yields

$$
\begin{aligned}
\left(\tilde{T}_{h} f\right)(\tilde{x})= & \left.\left(f_{1}\left(x_{1}, x_{2}\right)\right)(h / 4)\left(\tilde{x}_{3}-1 / 2\right)\left(r^{2}+\left(h\left(\tilde{x}_{3}-1 / 2\right)\right)^{2}\right)^{-1 / 2}\right|_{r=0} ^{r=\delta} \\
& -\left.\left(f_{2}\left(x_{1}, x_{2}\right)\right)(h / 4)\left(\tilde{x}_{3}+1 / 2\right)\left(r^{2}+\left(h\left(\tilde{x}_{3}+1 / 2\right)\right)^{2}\right)^{-1 / 2}\right|_{r=0} ^{r=\delta}+R_{7} .
\end{aligned}
$$

Again, the $r=\delta$ term goes in with the remainder, so as $h \rightarrow 0$, this converges to

$$
-1 / 4 f_{1}+1 / 4 f_{2}
$$

Step 2: Convergence of $\left\langle\tilde{T}_{h} f, g\right\rangle$ : Looking at the formula (3.6), we introduce the auxiliary operators $S_{1, h}, S_{2, h}$, and $S_{3, h}$, so that $\tilde{T}_{h}=S_{1, h}+S_{2, h}+S_{3, h}$. Here, $S_{1, h}$ is the integral over the top surface, $S_{2, h}$ is the integral over the bottom surface, and $S_{3, h}$ is the integral over the lateral surface. For $j \in\{1,2,3\}$, we will show that $\left\langle S_{j, h} f, g\right\rangle$ converges to $\left\langle S_{j, 0} f, g\right\rangle$, where $S_{j, 0}$ are the relevant pieces of $\tilde{T}_{0}$.

We begin with $S_{3, h}$, since this is the most intricate piece of the weak convergence argument. We need to prove

$$
h \int_{\partial \Omega_{h}} g(x) \int_{\partial \Omega \times\left[-\frac{1}{2}, \frac{1}{2}\right]} f_{3}(y) \partial_{\nu_{y}} \bar{\phi}(x, y) d \sigma_{y} d x \rightarrow 0 \quad \text { as } h \rightarrow 0 .
$$


We will consider this first for $x \in \Omega \times\left\{\frac{1}{2}\right\}$, and then we will consider $x$ on the other pieces of the boundary. Towards this end, we consider here

$$
I=h \int_{\Omega \times\left\{\frac{1}{2}\right\}} g_{1}(x)\left(\int_{\partial \Omega \times\left[-\frac{1}{2}, \frac{1}{2}\right]} f_{3}(y) \partial_{\nu_{y}} \bar{\phi}(x, y) d y\right) d x .
$$

We bound $f_{3}$ and $g_{1}$ by their infinity norms, and we change the order of integration:

$$
|I| \leq h\left|g_{1}\right|_{\infty}\left|f_{3}\right|_{\infty} \int_{\partial \Omega \times\left[-\frac{1}{2}, \frac{1}{2}\right]} \int_{\Omega \times\left\{\frac{1}{2}\right\}}\left|\partial_{\nu_{y}} \bar{\phi}(x, y)\right| d x d y .
$$

We rewrite the integral over the lateral boundary as being an arclength integral over $\partial \Omega$ and an integral with respect to $y_{3}$ :

$$
|I| \leq h\left|g_{1}\right|_{\infty}\left|f_{3}\right|_{\infty} \int_{-\frac{1}{2}}^{\frac{1}{2}} \int_{\partial \Omega} \int_{\Omega \times\left\{\frac{1}{2}\right\}}\left|\partial_{\nu_{y}} \bar{\phi}\left(x,\left(y_{1}(s), y_{2}(s), h \tilde{y}_{3}\right)\right)\right| d x d s d \tilde{y}_{3} .
$$

On the lateral boundary, the $y_{3}$ direction is tangential, so the normal vector is only in the $y_{1}$ and $y_{2}$ directions. Therefore,

$$
\left|\partial_{\nu_{y}} \bar{\phi}(x, y)\right| \leq\left|\frac{\partial \bar{\phi}}{\partial y_{1}}(x, y)\right|+\left|\frac{\partial \bar{\phi}}{\partial y_{2}}(x, y)\right| \text {. }
$$

Based on this, we write $I=I_{1}+I_{2}$, so that $I_{j}$ is the contribution from $\frac{\partial \bar{\phi}}{\partial y_{j}}$. We can calculate

$$
\begin{aligned}
& \frac{\partial \bar{\phi}}{\partial y_{1}}(x, y)=-\frac{1}{4 \pi} \frac{e^{-i k \sqrt{\left(x_{1}-y_{1}\right)^{2}+\left(x_{2}-y_{2}\right)^{2}+h^{2}\left(\tilde{x}_{3}-\tilde{y}_{3}\right)^{2}}}}{\left(\left(x_{1}-y_{1}\right)^{2}+\left(x_{2}-y_{2}\right)^{2}+h^{2}\left(\tilde{x}_{3}-\tilde{y}_{3}\right)^{2}\right)^{3 / 2}}\left(x_{1}-y_{1}\right)
\end{aligned}
$$

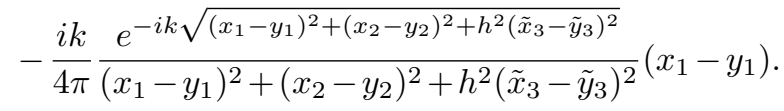

For a fixed $s$ and fixed $\tilde{y}_{3}$, we use polar coordinates for $x$. We have $x_{1}-y_{1}(s)=r \cos (\theta)$ and $x_{2}-y_{2}(s)=r \sin (\theta)$. We then have the following inequality:

$$
\left|\frac{\partial \bar{\phi}}{\partial y_{1}}(x, y)\right| \leq c \frac{r}{\left(r^{2}+h^{2}\left(\tilde{x}_{3}-\tilde{y}_{3}\right)^{2}\right)^{3 / 2}}+c \frac{r}{r^{2}+h^{2}\left(\tilde{x}_{3}-\tilde{y}_{3}\right)^{2}} .
$$

If we choose $R$ to be the diameter of $\Omega$, then $\Omega$ is a subset of the (two-dimensional) ball centered at $\left(y_{1}(s), y_{2}(s)\right)$ with radius $R$; therefore,

$$
\left|I_{1}\right| \leq c h\left|g_{1}\right|_{\infty}\left|f_{3}\right|_{\infty} \int_{-\frac{1}{2}}^{\frac{1}{2}} \int_{\partial \Omega} \int_{0}^{2 \pi} \int_{0}^{R} \frac{r^{2}}{\left(r^{2}+h^{2}\left(\tilde{x}_{3}-\tilde{y}_{3}\right)^{2}\right)^{3 / 2}}+\frac{r^{2}}{r^{2}+h^{2}\left(\tilde{x}_{3}-\tilde{y}_{3}\right)^{2}} d r d \theta d s d \tilde{y}_{3} .
$$

In this last integral, notice that the integrand does not depend on $s$ or $\theta$. Since $s$ and $\theta$ are both taken from a bounded interval, we can bound these integrals by a constant times the remaining integrals. We get

$$
\left|I_{1}\right| \leq c h\left|g_{1}\right|_{\infty}\left|f_{3}\right|_{\infty} \int_{-\frac{1}{2}}^{\frac{1}{2}} \int_{0}^{R} \frac{r^{2}}{\left(r^{2}+h^{2}\left(\tilde{x}_{3}-\tilde{y}_{3}\right)^{2}\right)^{3 / 2}}+\frac{r^{2}}{r^{2}+h^{2}\left(\tilde{x}_{3}-\tilde{y}_{3}\right)^{2}} d r d \tilde{y}_{3} .
$$


We have the following indefinite integral:

$$
\int \frac{r^{2}}{\left(r^{2}+\alpha^{2}\right)^{3 / 2}} d r=\ln \left(r+\sqrt{r^{2}+\alpha^{2}}\right)-\frac{r}{\sqrt{r^{2}+\alpha^{2}}}+c .
$$

Using this antiderivative, we have

$$
\left|I_{1}\right| \leq c h\left|f_{3}\right|_{\infty}\left|g_{1}\right|_{\infty} \int_{-\frac{1}{2}}^{\frac{1}{2}} 1+\left|\ln \left(h\left(\tilde{x}_{3}-\tilde{y}_{3}\right)\right)\right| d \tilde{y}_{3} .
$$

Since the natural logarithm is integrable, we conclude that

$$
\left|I_{1}\right| \leq \operatorname{ch}(|\ln (h)|+1)\left|f_{3}\right|_{\infty}\left|g_{1}\right|_{\infty} .
$$

Of course, we have the same bound for $I_{2}$. This implies

$$
|I| \leq \operatorname{ch}(|\ln (h)|+1)\left|f_{3}\right|_{\infty}\left|g_{1}\right|_{\infty}
$$

Clearly, then, $I$ goes to zero as $h$ goes to zero. If we had integrated over the bottom surface instead, we would find the same conclusion. Also, when $x \in \partial \Omega \times\left(-\frac{1}{2}, \frac{1}{2}\right)$, we see from the pointwise convergence argument that $S_{3, h} f(x)$ converges uniformly to zero. Taking all of this together, we have $\left\langle S_{3, h} f, g\right\rangle$ converges to zero as $h \rightarrow 0$.

We turn now to considering $S_{1, h}$. First, notice that if $x \in \Omega \times\left\{\frac{1}{2}\right\}$, then $S_{1, h} f(x)=$ 0 . Let $K \subseteq \Omega$ be a compact subset of $\Omega$, such that $K \cap \partial \Omega=\emptyset$. Then, by examining the pointwise convergence argument, we can see that $S_{1, h} f(x)$ converges uniformly for $x \in K \times\left\{-\frac{1}{2}\right\}$. More generally, for $x \in \Omega \times\left\{-\frac{1}{2}\right\}$, we have the following uniform bound:

$$
\begin{aligned}
\left|S_{1} f(x)\right| & \leq C \int_{\Omega \times\left\{\frac{1}{2}\right\}}\left|\partial_{y_{3}} \bar{\phi}(x, y)\right|\left|f_{1}(\tilde{y})\right| d \tilde{y} \\
& \leq C\left|f_{1}\right|_{\infty} \int_{\Omega} \frac{h}{\left(\left(x_{1}-y_{1}\right)^{2}+\left(x_{2}-y_{2}\right)^{2}+h^{2}\right)^{3 / 2}} d y_{1} d y_{2} \\
& \leq C\left|f_{1}\right|_{\infty} .
\end{aligned}
$$

This uniform bound implies that the integral

$$
\int_{(\Omega \backslash K) \times\left\{-\frac{1}{2}\right\}} g_{2}(x) \int_{\Omega \times\left\{\frac{1}{2}\right\}} \partial_{y_{3}} \bar{\phi}(x, y) f_{1}(y) d y d x
$$

can be made small by choosing $K$ appropriately. Furthermore, for $x \in \partial \Omega \times\left(-\frac{1}{2}, \frac{1}{2}\right)$, we see from the pointwise convergence argument that the convergence of $S_{1, h} f(x)$ is uniform. We have now established the convergence of $\left\langle S_{1, h} f, g\right\rangle$ as $h$ goes to zero. The weak convergence argument for $S_{2, h}$ is the same as for $S_{1, h}$. This completes the proof.

REMARK 3.1. The weak pointwise convergence of $\tilde{T}_{h}$ to $\tilde{T}_{0}$ automatically implies the weak pointwise convergence of the adjoints, that is,

$$
\left\langle\tilde{T}_{h}^{*} f, g\right\rangle \rightarrow\left\langle\tilde{T}_{0}^{*} f, g\right\rangle
$$

for any $f, g \in C_{P}^{0}$, where $\tilde{T}_{0}^{*}$ is the adjoint of $\tilde{T}_{0}$. 
In the boundary equation (2.17), the last two terms become

$$
\left(\frac{1}{2} I-T_{h}^{*}\right)\left(\left(n^{2}-1\right)^{-}(E \cdot \nu)^{-}\right)
$$

This motivates the following lemma, which will be useful in the next section.

Lemma 3.2. Let $T_{h}^{*}$ be given by (3.1) and let $T_{0}^{*}$ be its scaled pointwise limit as given in Theorem 3.1. For any fixed $h$, assume that $-k^{2}$ is not a Neumann eigenvalue for the Laplacian on $\Omega_{h}$. Then the operator

$$
\left(-\frac{1}{2} I+T_{h}^{*}\right)
$$

is invertible. However, the scaled limiting operator

$$
\left(-\frac{1}{2} I+\tilde{T}_{0}^{*}\right)
$$

is not invertible, and it has a null space consisting of all functions on $\partial S$ which have equal values on the top and bottom and are zero on the lateral boundary.

Proof. Recall that since for fixed $h, T_{h}^{*}$ is the dual of a double layer potential, it is well known that $-\frac{1}{2} I+T_{h}^{*}$ is Fredholm. Therefore, it is invertible if it has trivial null space. Suppose $\beta \in C^{0}\left(\partial \Omega_{h}\right)$ such that

$$
-\frac{1}{2} \beta+T_{h}^{*} \beta=0
$$

Define $w$ to be the single layer potential with moment $\beta$,

$$
w(x)=\int_{\partial \Omega_{h}} \phi(x, y) \beta(y) d \sigma_{y},
$$

defined for any $x \in \mathbb{R}^{3}$. By standard potential theory we have that the interior limit for the normal derivative of $w$ satisfies

$$
\left(\partial_{\nu} w\right)^{-}=-\frac{1}{2} \beta+T_{h}^{*} \beta=0,
$$

that $w$ satisfies the Helmholtz equation

$$
\Delta w+k^{2} w=0
$$

both in the interior $\Omega_{h}$ and the exterior $\mathbb{R}^{3} \backslash \Omega_{h}$, and we have that the jumps across the boundary of $\Omega_{h}$ are given by

$$
[w]=0
$$

and

$$
\left[\frac{\partial w}{\partial \nu}\right]=\beta
$$

Assuming $-k^{2}$ is not a Neumann eigenvalue for the Laplacian on $\Omega_{h}$, since $w$ satisfies (3.17), it must be identically zero inside of $\Omega_{h}$. Since the jump (3.18) is zero, this means that on the exterior, $w$ satisfies the Dirichlet problem for the Helmholtz 
equation with zero boundary data. So, $w$ must be identically zero on the exterior by Theorem 3.7 of [3]. Hence $w$ is identically zero on $\mathbb{R}^{3}$. Hence its normal derivative jump across $\partial \Omega_{h}$ must be zero, so from (3.19) we have $\beta=0$. Therefore we have that the null space is trivial and the operator $-\frac{1}{2} I+T_{h}^{*}$ is invertible.

For the limiting operator, the proof is simple algebra. Using the notation in the previous theorem, we calculate

$$
\left(-\frac{1}{2} I+\tilde{T}_{0}^{*}\right) f=\left(\begin{array}{ccc}
-\frac{1}{2} & \frac{1}{2} & -\frac{1}{4} R^{*} \\
\frac{1}{2} & -\frac{1}{2} & \frac{1}{4} R^{*} \\
0 & 0 & -\frac{1}{2}
\end{array}\right)\left(\begin{array}{l}
f_{1} \\
f_{2} \\
f_{3}
\end{array}\right),
$$

where $R^{*}$ is the adjoint of the restriction and extension operator, and its range is in the dual space to continuous functions on $\Omega$. One can calculate that for continuous $f_{3}$,

$$
R^{*} f_{3}=\left(\int_{-1 / 2}^{1 / 2} f_{3} d \tilde{x}_{3}\right) \delta_{\partial \Omega},
$$

a Dirac mass on the boundary multiplied by the $\tilde{x}_{3}$ average of $f_{3}$. We see directly that the null space consists of all $f$ such that $f_{1}=f_{2}$ and $f_{3}=0$.

\section{Limiting behavior of $E$}

Theorem 2.1 gives us a Lippman-Schwinger type of formulation for any Maxwell scatterer with regular enough boundary. In particular, when the index of refraction is of the specific form (2.2), it is useful for analyzing the behavior of the electric field as $h \rightarrow 0$. In this section we show two theorems about the asymptotic behavior of $E$ in this case. The first is that, on the lateral boundary of the domain, the interior limit of the normal component of the electric field, $(E \cdot \nu)^{-}$, goes to zero. The second result is that with some assumptions of uniform boundedness, the third component of the electric field, $E_{3}$, goes to zero with $h$. These two results together show that the high contrast structure does indeed "trap" the wave, in the sense that the flow out of the scatterer is small with respect to $h$.

Before stating these theorems, let us define some operators to more compactly describe the system (2.16). Set, for $f \in\left[L^{2}\left(\Omega_{h}\right)\right]^{3}$,

$$
\begin{gathered}
K^{h} f=\int_{\Omega_{h}} \phi(x, y)\left(\frac{\varepsilon_{0}(y)}{h}-1\right) f(y) d y \\
S^{h} f=\nabla_{x} \int_{\Omega_{h}} \phi(x, y) \frac{\nabla \varepsilon_{0}(y)}{\varepsilon_{0}(y)} \cdot f(y) d y \\
Q^{h} f=\int_{\partial \Omega \times(-h / 2, h / 2)} \nabla_{y} \phi(x, y)\left(\frac{\varepsilon_{0}(y)}{h}-1\right) f(y) \cdot \nu d \sigma_{y}, \\
-\int_{\Omega \times\{h / 2\}} \nabla_{y} \phi(x, y)\left(\frac{\varepsilon_{0}(y)}{h}-1\right) f_{3}(y) d y_{1} d y_{2} \\
\nabla_{y} \phi(x, y)\left(\frac{\varepsilon_{0}(y)}{h}-1\right) f_{3}(y) d y_{1} d y_{2},
\end{gathered}
$$


where $\nu=\left(\nu_{1}, \nu_{2}, 0\right)$ is the outward unit normal to the lateral boundary $L=\partial \Omega \times$ $(-h / 2, h / 2)$, and $f_{3}(y)$ is the third component of the vector-valued $f$. With this notation, $E$ satisfies

$$
E=E_{\mathbf{i}}+k^{2} K^{h} E+S^{h} E+L^{h} E+Q^{h} E,
$$

where $K^{h}$ is the operator coming from the Helmholtz-like term, $S^{h}$ is the operator coming from the smooth variation in $n$ in the interior, $L^{h}$ is the operator resulting from the lateral boundary of the object, and finally $Q^{h}$ is the operator coming from the top and bottom boundary of the object (combined). From [9], we know that

$$
\left\|K^{h} f\right\|_{\infty} \leq C\|f\|_{\infty}
$$

while from [1] we have

$$
\left\|S^{h} f\right\|_{\infty} \leq C h|\log h|\|f\|_{\infty},
$$

where in both cases $C$ is independent of $h$. Similar to $C_{P}^{0}$, define the piecewise $C^{\alpha}$ space on the scaled boundary:

$$
C_{P}^{\alpha}=C^{\alpha}\left(\bar{\Omega} \times\left\{\frac{1}{2}\right\}\right) \oplus C^{\alpha}\left(\bar{\Omega} \times\left\{\frac{-1}{2}\right\}\right) \oplus C^{\alpha}\left(\partial \Omega \times\left[-\frac{1}{2}, \frac{1}{2}\right]\right) .
$$

Also, we represent all functions in the scaled variables (3.2) with a tilde, that is,

$$
\tilde{E}(\tilde{y})=E(y) .
$$

Theorem 4.1. Let $E$ solve (2.16) with $n^{2}(x)$ defined by (2.2), and let $(E \cdot \nu)^{-}$be the interior limit of $E \cdot \nu$ on the boundary. Assume that $\varepsilon_{0} \in C^{0}\left(\bar{\Omega}_{h}\right)$ is such that $\varepsilon_{0}$ is bounded away from zero. Assume that $E$ is bounded in $C^{0}\left(\overline{\Omega_{h}}\right)$ independently of $h$, and that $(\tilde{E} \cdot \nu)^{-}$is bounded in $C_{P}^{\alpha}$ independently of $h$, for some $\alpha>0$. Then we have that

$$
(\tilde{E} \cdot \nu)^{-} \rightarrow 0,
$$

uniformly on the lateral boundary $\partial \Omega \times(-1 / 2,1 / 2)$, as $h \rightarrow 0$.

Proof. Consider (2.17) with $n^{2}(x)=\varepsilon_{0}(x) / h$ in the interior of $\Omega_{h}$. Multiplying everything by $h$ and collecting what appear to be $O(1)$ terms, we have that

$$
\frac{1}{2} \varepsilon_{0}(E \cdot \nu)^{-}-\int_{\partial \Omega_{h}} \partial_{\nu_{x}} \phi \varepsilon_{0}\left(E \cdot \nu_{y}\right)^{-} d \sigma_{y}=h\left(E_{i} \cdot \nu-\frac{3}{2}(E \cdot \nu)^{-}+k^{2}\left(K^{h} E\right) \cdot \nu+\left(S^{h} E\right) \cdot \nu\right) .
$$

Note that by the uniform boundedness assumption on $E$ and bounds (4.6) and (4.7), the right hand side is continuous on $\Omega_{h}$ and $O(h)$ in $L^{\infty}\left(\partial \Omega_{h}\right)$. Consider, for $x \in \partial \Omega_{h}$,

$$
\psi_{h}(x)=\varepsilon_{0}(x)(E \cdot \nu)^{-}(x) .
$$

Then $\psi_{h}(x)$ satisfies the Fredholm equation

$$
-\frac{1}{2} \psi_{h}+T_{h}^{*} \psi_{h}=e_{h},
$$

where $T_{h}^{*}$ is the operator (3.1), the unscaled version of the dual of operator from Theorem 3.1. Also, $e_{h}$ is the negative of the right hand side of (4.9) above. Rescaling in $x_{3}$, we have

$$
\left(-\frac{1}{2} I+\tilde{T}_{h}^{*}\right) \tilde{\psi}_{h}=\tilde{e}_{h} .
$$


Note that by our assumptions, the $\left\{\tilde{\psi}_{h}\right\}$ are uniformly bounded in $C^{\alpha}$ separately on each piece of $\partial S$. By the Arzela-Ascoli Theorem, there exists a subsequence with a uniform limit; call this limit $\tilde{\psi}_{0}$. For any $f \in C_{P}^{0}$,

$$
\left\langle\left(-\frac{1}{2} I+\tilde{T}_{h}^{*}\right) \tilde{\psi}_{h}, f\right\rangle=\left\langle\tilde{e}_{h}, f\right\rangle
$$

where the inner product is the usual one for $L^{2}(\partial S)$. By taking the dual of the operator, this gives

$$
\left\langle\tilde{\psi}_{h},\left(-\frac{1}{2} I+\tilde{T}_{h}\right) f\right\rangle=\left\langle\tilde{e}_{h}, f\right\rangle
$$

where $\tilde{T}_{h}$ is the dual to $\tilde{T}_{h}^{*}$, as in Theorem 3.1. Also from this theorem we know that $\tilde{T}_{h}$ converges pointwise weakly to $\tilde{T}_{0}$. By adding and subtracting we have

$$
\begin{aligned}
\left\langle\tilde{\psi}_{h},\left(-\frac{1}{2} I+\tilde{T}_{0}\right) f\right\rangle & =\left\langle\tilde{\psi}_{h},\left(\tilde{T}_{0}-\tilde{T}_{h}\right) f\right\rangle+\left\langle\tilde{e}_{h}, f\right\rangle \\
& =\left\langle\tilde{\psi}_{h}-\tilde{\psi}_{0},\left(\tilde{T}_{0}-\tilde{T}_{h}\right) f\right\rangle+\left\langle\tilde{\psi}_{0},\left(\tilde{T}_{0}-\tilde{T}_{h}\right) f\right\rangle+\left\langle\tilde{e}_{h}, f\right\rangle
\end{aligned}
$$

Notice that since $\tilde{T}_{h} f$ converges weakly, the Principle of Uniform Boundedness implies that it is bounded uniformly with respect to $h$. This can also be seen directly by the weak convergence proof of the operators. Therefore, if we let $h \rightarrow 0$, everything on the right-hand side goes to zero and we have

$$
\left\langle\tilde{\psi}_{0},\left(-\frac{1}{2} I+\tilde{T}_{0}\right) f\right\rangle=0
$$

which implies that

$$
\left\langle\left(-\frac{1}{2} I+\tilde{T}_{0}^{*}\right) \tilde{\psi}_{0}, f\right\rangle=0
$$

for any $f \in C_{P}^{0}$. Hence $\tilde{\psi}_{0}$ lies in the null space of $\left(-\frac{1}{2} I+\tilde{T}_{0}^{*}\right)$. From Lemma 3.1 this implies that $\tilde{\psi}_{0}$ is zero on the lateral boundary (and equal on the top and bottom). Therefore any sequence of $\{\tilde{E} \cdot \nu\}$ has a subsequence which converges uniformly to zero on the lateral boundary; this implies the conclusion of the theorem.

TheOrem 4.2. Let $E_{3} \in C^{\alpha}\left(\Omega_{h}\right)$ for some $0<\alpha \leq 1$ be the solution to (2.16) such that

$$
\left\|E_{3}\right\|_{C^{\alpha}\left(\Omega_{h}\right)} \leq M_{\alpha}
$$

where $M_{\alpha}$ is independent of $h$. Then, pointwise for $x \in \Omega_{h}$, there exists $C$ depending on $\alpha$ and possibly $x$ such that, for $\alpha<1$, we have

$$
\left|E_{3}(x)\right| \leq C M_{\alpha} h^{\alpha}
$$

and for $\alpha=1$ we have

$$
\left|E_{3}(x)\right| \leq C M_{1} h|\log h|
$$

where $C$ is independent of $h$. 
Proof. We will examine the term in (4.5) which we expect will blow up and multiply it by $h$. We first fix $x_{3}=0$ and compute that

$$
\begin{aligned}
h\left(Q_{h} E\right)_{3}=\int_{\Omega} & \left.\frac{\partial \phi}{\partial y_{3}}(x, y)\left(n_{0}(y)-h\right) E_{3}(y)\right|_{y_{3}=h / 2} d y_{1} d y_{2} \\
& \quad-\left.\int_{\Omega} \frac{\partial \phi}{\partial y_{3}}(x, y)\left(n_{0}(y)-h\right) E_{3}(y)\right|_{y_{3}=-h / 2} d y_{1} d y_{2} \\
= & \left.n_{0}(x)-h\right) E_{3}(x) \int_{\Omega}\left(\left.\frac{\partial \phi}{\partial y_{3}}(x, y)\right|_{y_{3}=h / 2}-\left.\frac{\partial \phi}{\partial y_{3}}(x, y)\right|_{y_{3}=-h / 2}\right) d y_{1} d y_{2} \\
& +\left.\int_{\Omega} \frac{\partial \phi}{\partial y_{3}}(x, y)\left[\left(n_{0}(y)-h\right) E_{3}(y)-\left(n_{0}(x)-h\right) E_{3}(x)\right]\right|_{y_{3}=h / 2} d y_{1} d y_{2} \\
& +\left.\int_{\Omega} \frac{\partial \phi}{\partial y_{3}}(x, y)\left[\left(n_{0}(y)-h\right) E_{3}(y)-\left(n_{0}(x)-h\right) E_{3}(x)\right]\right|_{y_{3}=-h / 2} d y_{1} d y_{2} .
\end{aligned}
$$

From the Hölder condition on $E$ and the smoothness assumption on $n_{0}$,

$$
\left|\left(n_{0}(y)-h\right) E_{3}(y)-\left(n_{0}(x)-h\right) E_{3}(x)\right| \leq C_{\alpha}|x-y|^{\alpha} .
$$

This implies

$$
\begin{aligned}
& \left.\int_{\Omega} \frac{\partial \phi}{\partial y_{3}}(x, y)\right|_{y_{3}=h / 2}\left[\left(n_{0}(y)-h\right) E_{3}(y)-\left(n_{0}(x)-h\right) E_{3}(x)\right] d y_{1} d y_{2} \\
\leq & C \int_{\Omega}\left|\frac{\partial \phi}{\partial y_{3}}(x, y)\right|_{y_{3}=h / 2}|| x-\left.y\right|^{\alpha} d y_{1} d y_{2} .
\end{aligned}
$$

If we define $r$ to be the two-dimensional distance

$$
r=\left|\left(x_{1}, x_{2}\right)-\left(y_{1}, y_{2}\right)\right|,
$$

we see that

$$
\left|\frac{\partial \phi}{\partial y_{3}}(x, y)\right|_{y_{3}=h / 2} \mid \leq C h \frac{1}{\left(r^{2}+h^{2}\right)^{3 / 2}},
$$

so for some $C$ independent of $h$, we have (letting $R$ be sufficiently large)

$$
\begin{aligned}
\int_{\Omega}\left|\frac{\partial \phi}{\partial y_{3}}(x, y)\right|_{y_{3}=h / 2}|| x-\left.y\right|^{\alpha} d y_{1} d y_{2} & \leq C h \int_{\Omega} \frac{r^{\alpha}}{\left(r^{2}+h^{2}\right)^{3 / 2}} d y_{1} d y_{2} \\
& \leq C h \int_{0}^{R} \frac{r^{1+\alpha}}{\left(r^{2}+h^{2}\right)^{3 / 2}} d r .
\end{aligned}
$$

A computation yields that for $0<\alpha<1$,

$$
\int_{0}^{R} \frac{r^{1+\alpha}}{\left(r^{2}+h^{2}\right)^{3 / 2}} d r \leq C h^{\alpha-1}+O(1)
$$

and for $\alpha=1$,

$$
\int_{0}^{R} \frac{r^{2}}{\left(r^{2}+h^{2}\right)^{3 / 2}} d r \leq C|\log h|+O(1)
$$


The same bound also holds for the last term in the right hand side of (4.18). This then gives that, for $0<\alpha<1$,

$$
h\left(Q_{h} E\right)_{3}=\left(n_{0}(x)-h\right) E_{3}(x) \int_{\Omega}\left(\left.\frac{\partial \phi}{\partial y_{3}}(x, y)\right|_{y_{3}=h / 2}-\left.\frac{\partial \phi}{\partial y_{3}}(x, y)\right|_{y_{3}=-h / 2}\right) d y_{1} d y_{2}+O\left(h^{\alpha}\right) .
$$

Set

$$
I_{h}=\int_{\Omega}\left(\left.\frac{\partial \phi}{\partial y_{3}}(x, y)\right|_{y_{3}=h / 2}-\left.\frac{\partial \phi}{\partial y_{3}}(x, y)\right|_{y_{3}=-h / 2}\right) d y_{1} d y_{2}
$$

Using the above in the third component of (4.5) we have

$$
\left(n_{0}(x)-h\right) E_{3}(x) I_{h}=h E_{3}-h\left(E_{\mathbf{i}}\right)_{3}-h k^{2}\left(T^{h} E\right)_{3}-h\left(S^{h} E\right)_{3}+h\left(L^{h} E\right)+O\left(h^{\alpha}\right) .
$$

Now, we will show that $I_{h}$ is actually bounded from below away from zero. We recall the definition of $\phi$ :

$$
\phi(x, y)=\frac{1}{4 \pi} \frac{e^{i k|x-y|}}{|x-y|} .
$$

We take the derivative of this with respect to $y_{3}$ to get

$$
\frac{\partial \phi(x, y)}{\partial y_{3}}=\frac{1}{4 \pi} \frac{e^{i k|x-y|}}{|x-y|^{3}}\left(x_{3}-y_{3}\right)-\frac{i k}{4 \pi} \frac{e^{i k|x-y|}}{|x-y|^{2}}\left(x_{3}-y_{3}\right) .
$$

Next, we set $x_{3}=0$ so that

$$
\left.\frac{\partial \phi(x, y)}{\partial y_{3}}\right|_{x_{3}=0}=-\left.\frac{y_{3}}{4 \pi} \frac{e^{i k|x-y|}}{|x-y|^{3}}\right|_{x_{3}=0}+\left.\frac{i k y_{3}}{4 \pi} \frac{e^{i k|x-y|}}{|x-y|^{2}}\right|_{x_{3}=0} .
$$

We let $r=\sqrt{\left(x_{1}-y_{1}\right)^{2}+\left(x_{2}-y_{2}\right)^{2}}$, plug in $y_{3}=h / 2$ and $y_{3}=-h / 2$, and subtract to obtain

$$
\left.\frac{\partial \phi(x, y)}{\partial y_{3}}\right|_{\substack{x_{3}=0, y_{3}=h / 2}}-\left.\frac{\partial \phi(x, y)}{\partial y_{3}}\right|_{\substack{x_{3}=0, y_{3}=-h / 2}}=-\frac{h}{4 \pi} \frac{e^{i k\left(r^{2}+h^{2} / 4\right)}}{\left(r^{2}+h^{2} / 4\right)^{3 / 2}}+\frac{i k h}{4 \pi} \frac{e^{i k\left(r^{2}+h^{2} / 4\right)}}{\left(r^{2}+h^{2} / 4\right)} .
$$

Rewriting this and adding and subtracting 1 from the first exponential we have

$$
\begin{aligned}
& \left.\frac{\partial \phi(x, y)}{\partial y_{3}}\right|_{\substack{x_{3}=0, y_{3}=h / 2}}-\left.\frac{\partial \phi(x, y)}{\partial y_{3}}\right|_{\substack{x_{3}=0, y_{3}=-h / 2}} \\
= & -\frac{h}{4 \pi} \frac{1}{\left(r^{2}+h^{2} / 4\right)^{3 / 2}}+\frac{i k h}{4 \pi} \frac{e^{i k\left(r^{2}+h^{2} / 4\right)}}{\left(r^{2}+h^{2} / 4\right)}-\frac{h}{4 \pi} \frac{\left(e^{i k\left(r^{2}+h^{2} / 4\right)}-1\right)}{\left(r^{2}+h^{2} / 4\right)^{3 / 2}} .
\end{aligned}
$$

Since $\left(x_{1}, x_{2}\right)$ is in the interior of $\Omega$, there exists $\delta>0$ such that $B_{\delta}\left(x_{1}, x_{2}\right) \subseteq \Omega$. We therefore write

$$
I_{h}\left(x_{1}, x_{2}, 0\right)=A_{1}+A_{2}+A_{3}+\int_{\Omega \backslash B_{\delta}\left(x_{1}, x_{2}\right)}\left(\left.\frac{\partial \phi(x, y)}{\partial y_{3}}\right|_{\substack{x_{3}=0, y_{3}=h / 2}}-\left.\frac{\partial \phi(x, y)}{\partial y_{3}}\right|_{\substack{x_{3}=0, y_{3}=-h / 2}}\right) d y_{1} d y_{2} ;
$$

we write it in this fashion since we have three terms on the right-hand side of (4.21), and we write the region of integration as $\Omega=B_{\delta}\left(x_{1}, x_{2}\right) \bigcup\left(\Omega \backslash B_{\delta}\left(x_{1}, x_{2}\right)\right)$. 
Using (4.21), we can see that the integral over the complement of the ball goes to zero with $h$, that is

$$
\int_{\Omega \backslash B_{\delta}\left(x_{1}, x_{2}\right)}\left(\left.\frac{\partial \phi(x, y)}{\partial y_{3}}\right|_{\substack{x_{3}=0, y_{3}=h / 2}}-\left.\frac{\partial \phi(x, y)}{\partial y_{3}}\right|_{\substack{x_{3}=0, y_{3}=-h / 2}}\right) d y_{1} d y_{2}=O(h) .
$$

We can bound $A_{1}$ by switching to polar coordinates as follows:

$$
\begin{aligned}
A_{1}=-\int_{B_{\delta}\left(x_{1}, x_{2}\right)} \frac{h}{4 \pi} \frac{1}{\left(r^{2}+h^{2} / 4\right)^{3 / 2}} d y_{1} d y_{2} & =-\frac{h}{2} \int_{0}^{\delta} \frac{r}{\left(r^{2}+h^{2} / 4\right)^{3 / 2}} d r \\
& =\left.\frac{h}{2} \frac{1}{\sqrt{r^{2}+h^{2} / 4}}\right|_{0} ^{\delta}=-1+O(h) .
\end{aligned}
$$

For $A_{2}$ we start by stating its definition:

$$
A_{2}=\int_{B_{\delta}\left(x_{1}, x_{2}\right)} \frac{i k h}{4 \pi} \frac{e^{i k\left(r^{2}+h^{2} / 4\right)}}{r^{2}+h^{2} / 4} d y_{1} d y_{2} .
$$

We switch to polar coordinates, and we bound the absolute value of this as

$$
\left|A_{2}\right| \leq \frac{k h}{2} \int_{0}^{\delta} \frac{r}{r^{2}+h^{2} / 4} d r=\left.\frac{k h}{4} \ln \left(r^{2}+h^{2} / 4\right)\right|_{0} ^{\delta}=O(h \ln (h)) .
$$

We need finally to bound $A_{3}$. The definition of $A_{3}$ is

$$
A_{3}=-\int_{B_{\delta}\left(x_{1}, x_{2}\right)} \frac{h}{4 \pi} \frac{\left(e^{i k\left(r^{2}+h^{2} / 4\right)}-1\right)}{\left(r^{2}+h^{2} / 4\right)^{3 / 2}} d y_{1} d y_{2} .
$$

By either the Cauchy or Lagrange form of the remainder in Taylor's Theorem, there exists a constant, $C$, such that $\left|e^{i k\left(r^{2}+h^{2} / 4\right)}-1\right| \leq C\left(r^{2}+h^{2} / 4\right)$. We again use polar coordinates. We estimate the absolute value of $A_{3}$ as

$$
\left|A_{3}\right| \leq \frac{C h}{2} \int_{0}^{\delta} \frac{r}{\left(r^{2}+h^{2}\right)^{1 / 2}} d r=O(h) .
$$

Taking these calculations together, we have shown that

$$
I_{h}\left(x_{1}, x_{2}, 0\right)=-1+O(h \ln (h)) .
$$

Hence from (4.20) we have that for $0<\alpha<1$

$$
n_{0}(x) E_{3}\left(x_{1}, x_{2}, 0\right)=O\left(h^{\alpha}\right)
$$

and for $\alpha=1$

$$
n_{0}(x) E_{3}\left(x_{1}, x_{2}, 0\right)=O(h \log h) .
$$

Using the $C^{\alpha}$ boundedness of $E_{3}$, the same bounds hold on all of $\Omega_{h}$. 


\section{Discussion}

We have derived and used the formulation in Theorem 2.1 to analyze the behavior of a thin high contrast dielectric. This formulation treats any jumps in the material with a surface integral, and we have shown that the surface terms force the normal component of the electric field to go to zero on the boundary of the thin high contrast domain, so they are indeed playing a crucial role. Here we discuss the implications for the limiting equations and further asymptotic expansions in $h$. If we assume that

$$
E(y)=E^{(0)}\left(y_{1}, y_{2}\right)+h E^{(1)}(y)+O\left(h^{2}\right),
$$

and we plug this into (2.16) and let $h \rightarrow 0$, then from [9] we know that the first integral term becomes

$$
k^{2} \int_{\Omega} \phi\left(\left(x_{1}, x_{2}, 0\right),\left(y_{1}, y_{2}, 0\right)\right) \varepsilon_{0}\left(y_{1}, y_{2}\right) E^{(0)}\left(y_{1}, y_{2}\right) d y_{1} d y_{2},
$$

and from [1] we know that the second integral term is $O(h \log h)$, so it disappears in the highest order limit. For the surface integral term,

$$
\int_{\partial \Omega_{h}} \nabla_{y} \phi(x, y)\left(\frac{\varepsilon_{0}(y)}{h}-1\right)(E(y) \cdot \nu)^{-} d \sigma_{y},
$$

the situation is more complicated. This term has of course three vector components, and in each of these components we have terms corresponding to the top, bottom, and lateral sides of the scatterer. In the first two components, the integral over the top and bottom parts of the boundary cancel with each other in the limit (this will be described in detail in a forthcoming work). The limit of the first two components of (5.1) therefore correspond to an integral over the lateral side, which becomes the curve integral

$$
\int_{\partial \Omega} \nabla_{y} \phi(x, y) \varepsilon_{0} E^{(0)} \cdot \nu d s_{y} .
$$

If $x$ is in the interior of $\Omega$, this term has no singularity, and by Theorem 4.1 it should disappear to first order, yielding the same limiting equations as in the Helmholtz and smooth cases [1], [9]. The limit in the first two components became nice because there was cancellation between the top and bottom parts of the surface integral as they get close to each other. However, for the third component, the top and bottom terms are the same sign and do not cancel in the limit, and get more singular as they approach; it is on the order of $E_{3} / h$. This is another way to see that $E_{3}$ goes to zero in the interior of the scatterer, since it allows the equations to balance. Hence the analysis (formally) yields the limiting system

$$
\begin{aligned}
& E_{1}^{(0)}\left(x_{1}, x_{2}\right)=\left(E_{\mathbf{i}}\right)_{1}\left(x_{1}, x_{2}, 0\right)+k^{2} \int_{\Omega} \phi \varepsilon_{0} E_{1}^{(0)}, \\
& E_{2}^{(0)}\left(x_{1}, x_{2}\right)=\left(E_{\mathbf{i}}\right)_{2}\left(x_{1}, x_{2}, 0\right)+k^{2} \int_{\Omega} \phi \varepsilon_{0} E_{2}^{(0)}, \\
& E_{3}^{(0)}\left(x_{2}, x_{2}\right)=0 .
\end{aligned}
$$

We propose the following first-order approximation to the field anywhere in $\mathbb{R}^{3}$ : solve the above two dimensional system for $E^{(0)}$, and insert this solution $E^{(0)}$ into the right hand side of (2.6) to compute the field elsewhere. This should be very efficient, and 
much more accurate than the commonly used Born approximation of just inserting the incident wave $E_{\mathbf{i}}$. We have indeed shown that (under certain regularity assumptions on $E$ ), the perpendicular component of $E$ to the slab, $E_{3}$, goes to zero inside the slab. This dimensionally reduced system will also be useful for approximating resonances as in [5], and for inversion methods such as in [11].

We have also shown here that on the lateral boundary to the slab, the interior normal component $(E \cdot \nu)^{-} \rightarrow 0$ in Theorem 4.1. This normal component to $\partial \Omega$ now involves a combination of $E_{1}$ and $E_{2}$. However, the solution to (5.3),(5.4) is unique (see [9]), and cannot in general satisfy that the normal component on $\partial \Omega$ is zero for arbitrary $E_{\mathbf{i}}$. This implies that any convergence of $E \rightarrow E^{(0)}$ can not be uniform in general: some sort of boundary layer must exist near $\partial \Omega$. This makes sense, since the kernel in (5.2) becomes singular as $x$ approaches the boundary of $\Omega$, and this term can no longer be discarded.

We now comment on how the results here relate to the previous results in [9] and [1]. The former only considered a scalar Helmholtz system, and there the first-order limiting system for the polarized field is the same as (5.3), and the convergence is uniform and $O(h)$. In [1], full Maxwell is considered, but there the squared index of refraction is assumed to be everywhere $C^{2}$. In particular, the effects of material jumps between $\Omega_{h}$ and the background are not considered. Our analysis here includes jumps, such as holes in $\Omega$, and no smoothing at the edge is required. It implies that, up to first order in the interior of $\Omega$, one gets the same limiting system for the first two components, but the surface term does force a different limit (namely zero) for the third component $E_{3}$. The surface term also plays a role in boundary behavior and will be crucial for deriving accurate corrections. Further asymptotic analysis of the boundary and the next order corrections will be the subject of a forthcoming paper.

Acknowledgment. D.M. Ambrose was supported by the National Science Foundation under grants DMS-1008387 and DMS-1016267. S. Moskow was supported by the National Science Foundation under grant DMS-1108858.

\section{REFERENCES}

[1] H. Ammari, H. Kang, and F. Santosa, Scattering of electromagnetic waves by thin dielectric planar structures, SIAM J. Math. Anal., 38, 1329-1342, 2006.

[2] G. Bouchitté, Analyse limite de la diffraction d'ondes électromagnétiques par une structure mince, C.R. Acad. Sci. Paris Sér. II Méc. Phys. Chim. Sci. Univers Sci. Terre, 311, 51-56, 1990.

[3] D. Colton and R. Kress, Inverse Acoustic and Electromagnetic Scattering Theory, Appl. Math. Sci., Springer, 93, 1998.

[4] G.B. Folland, Introduction to Partial Differential Equations, Princeton University Press, Princeton, N.J., 1976.

[5] J. Gopalakrishnan, S. Moskow, and F. Santosa, Asymptotic and numerical techniques for resonances of thin photonic structures, SIAM J. Appl. Math., 69, 37-63, 2008.

[6] A. Kirsch, An integral equation for Maxwell's equations in a layered medium with an application to the factorization method, J. Int. Eqs. Appl., 19, 333-358, 2007.

[7] A. Kirsch and N. Grinberg, The Factorization Method for Inverse Problems, Oxford Lecture Series in Mathematics and its Applications, Oxford University Press, Oxford, 36, 2008.

[8] A. Kirsch and A. Lechleiter, The operator equations of Lippmann-Schwinger type for acoustic and electromagnetic scattering problems in $L^{2}$, Appl. Anal., 88, 807-830, 2009.

[9] S. Moskow, F. Santosa, and J. Zhang, An approximate method for scattering by thin structures, SIAM J. Appl. Math., 66, 187-205, 2005.

[10] J.H. Richmond, Scattering by thin dielectric strips, IEEE Trans. Ant. Prop., 33, 64-68, 1985.

[11] N. Zeev and F. Cakoni, The identification of thin dielectric objects from far field or near field scattering data, SIAM J. Appl. Math., 69, 1024-1042, 2009. 